УДК 517.5

\author{
V. M. Dilnyi, T. I. HishCHAK
}

\title{
ON SPLITTING FUNCTIONS IN PALEY-WIENER SPACE
}

V. M. Dilnyi, T. I. Hishchak. On splitting functions in Paley-Wiener space, Mat. Stud. 45 (2016), 137-148.

We consider the problem on splitting functions in the Paley-Wiener space into the sum of two ones, each being "large" only in their domain.

Decomposition problems in spaces of analytic functions is concidered by many mathematicians. R. S. Yulmukhametov in [1], [2] considered the problem on splitting of function in Paley Wiener space into the product of two functions. Yu. I. Lyubarskii in [3] studied the splitting of functions with a triangle indicator diagram. I. E. Chyzhykov in [4] studied similar issues in terms of subharmonic functions. V. M. Dilnyi in [5] consider the problem about decomposition of a function in the Paley-Wiener space into the sum of two functions, each being "large" only on half-plane.

We denote by $W_{\sigma}^{p}, \sigma>0$, the Paley-Wiener space, i.e., the space of entire functions $f$ of exponential type $\leq \sigma$ belonging to $L^{p}(\mathbb{R})$. The space $W_{\sigma}^{p}$ can be defined (see [6, p. 663]) as the space of entire functions satisfying the condition

$$
\sup _{\varphi \in(0 ; 2 \pi)}\left\{\int_{0}^{+\infty}\left|f\left(r e^{i \varphi}\right)\right|^{p} e^{-p \sigma r|\sin \varphi|} d r\right\}^{\frac{1}{p}}<+\infty .
$$

The following statement plays a fundamental role in the theory of Paley-Wiener spaces ([2]).

Theorem. (Paley-Wiener) Space $f \in W_{\sigma}^{2}$ coincides with the space of functions represented as

$$
f(z)=\frac{1}{\sqrt{2 \pi}} \int_{-\sigma}^{\sigma} \varphi(i t) e^{i t z} d t, \varphi \in L^{2}(-i \sigma ; i \sigma) .
$$

Analogical statements are also known for the cases $1<p<2$ and $p=1$. For the case $p=1$ an analogue is proved by G. Z. Ber ([7]). In another form the criterion for belonging to $W_{\sigma}^{1}$ is obtained by R. Boas ([8, p. 106]) and other.

Theorem. ([7]) The space $f \in W_{\sigma}^{1}$ coincides with the space of functions represented by (1), where

$$
\varphi(t)=\sum_{k=-\infty}^{+\infty} c_{k} e^{\frac{-i k \pi t}{\sigma}}, \quad\left(c_{k}\right) \in l^{1}
$$

2010 Mathematics Subject Classification: 30D15, 30H10.

Keywords: Paley-Wiener space; angular boundary values; splitting. doi:10.15330/ms.45.2.137-148

(C) V. M. Dilnyi, T. I. Hishchak, 2016 
and

$$
\sum_{m=-\infty}^{+\infty}\left|\sum_{k=-\infty}^{+\infty}(-1)^{k+m} c_{k+m} \frac{k}{1+k^{2}}\right|<+\infty .
$$

Let $E^{p}[\mathbb{C}(\alpha ; \beta)], 0<\beta-\alpha<2 \pi, 1 \leq p<+\infty$, be the space of analytic functions $f$ in $\mathbb{C}(\alpha ; \beta)=\{z: \alpha<\arg z<\beta\}$ such that

$$
\sup _{\alpha<\varphi<\beta}\left\{\int_{0}^{+\infty}\left|f\left(r e^{i \varphi}\right)\right| d r\right\}<+\infty .
$$

Almost everywhere on $\partial \mathbb{C}(\alpha ; \beta)$, functions $f \in E^{p}[\mathbb{C}(\alpha ; \beta)]([9])$ have angular boundary values (we denote it by $f$ ) and $f \in L^{p}[\mathbb{C}(\alpha ; \beta)]$.

Problem. Whether each function $f \in W_{\sigma}^{1}$ admits a splitting $f=\chi+\mu$, where functions $\chi$ and $\mu$ are analytic in $\mathbb{C}_{+}=\{z: \operatorname{Re} z>0\}$, and $\chi \in E^{1}\left[\mathbb{C}\left(0 ; \frac{\pi}{2}\right)\right], \mu \in E^{1}\left[\mathbb{C}\left(-\frac{\pi}{2} ; 0\right)\right]$ ?

This problem is generated by studies of completeness of functions ([10]) and considered in [5]. Above problem is interesting in the theory of integral operators and the shift operator. We offer a new way of solving above problem other than in [5].

We find representations in the form of a function $\chi$

$$
\chi(z)=\chi_{1}(z)+i \chi_{2}(-i z)
$$

where

$$
\chi_{1}(z)=\frac{1}{\sqrt{2 \pi}} \int_{0}^{\sigma} \varphi(t) e^{i t z} d t, \quad \chi_{2}(z)=-\frac{1}{\sqrt{2 \pi}} \int_{-\sigma}^{0} \varphi(t) e^{i t z} d t .
$$

We write $\mu=f-\chi$, and understand that the function $\chi$ as a solution of the problem.

We obtain the following statement.

Theorem 1. Let $f \in W_{\sigma}^{1}$. The function $\chi$ defined by (3) is a solution of Problem if and only if both of the following conditions are fulfilled

$$
\begin{aligned}
& \sum_{m=1}^{+\infty}\left|\sum_{k=-\infty}^{+\infty} c_{k} \frac{k}{\left(m-\frac{i}{2}-k\right)\left(m-\frac{i}{2}-i k\right)}\right|<+\infty, \\
& \sum_{m=1}^{+\infty}\left|\sum_{k=-\infty}^{+\infty} c_{k} \frac{k}{\left(m+\frac{i}{2}+i k\right)\left(m+\frac{i}{2}-k\right)}\right|<+\infty .
\end{aligned}
$$

Example. Let $\varphi(t)=|t|-\sigma$. It is clear that $f(z)=\frac{\sqrt{2}}{\sqrt{\pi} z^{2}}(\cos \sigma z-1)$. Then

$$
\begin{aligned}
& \chi_{1}(z)=\frac{1}{\sqrt{2 \pi}} \int_{0}^{\sigma}(|t|-\sigma) e^{i t z} d t=\frac{1}{\sqrt{2 \pi}}\left(\frac{e^{i \sigma z}}{z^{2}}-\frac{1}{z^{2}}+\frac{\sigma}{i z}\right), \\
& \chi_{2}(-i z)=\frac{1}{\sqrt{2 \pi}} \int_{-\sigma}^{0}(|t|-\sigma) e^{t z} d t=\frac{1}{\sqrt{2 \pi}}\left(\frac{\sigma}{z}-\frac{1}{z^{2}}+\frac{e^{-\sigma z}}{z^{2}}\right) .
\end{aligned}
$$

Hence

$$
\chi(z)=\chi_{1}(z)+i \chi_{2}(-i z)=\frac{1}{\sqrt{2 \pi} z^{2}}\left(e^{i \sigma z}+i e^{-\sigma z}-1-i\right)
$$

and $\mu(z)=\frac{1}{\sqrt{2 \pi} z^{2}}\left(e^{-i \sigma z}-i e^{-\sigma z}-1+i\right)$. 
To prove Theorem 1, we need several lemmas.

Lemma 1. Let $f \in W_{\sigma}^{1}$. Then $\chi\left(x-i \frac{\pi}{2 \sigma}\right) \in L^{1}\left(\frac{\pi}{\sigma} ;+\infty\right)$ if and only if

$$
\int_{\frac{\pi}{\sigma}}^{+\infty}\left|\sum_{k=-\infty}^{+\infty} c_{k} \frac{k}{\left(x-\frac{\pi}{\sigma}\left(\frac{i}{2}+k\right)\right)\left(x-i \frac{\pi}{\sigma}\left(\frac{1}{2}+k\right)\right)}\right| d x<+\infty
$$

where coefficients $c_{k}$ defined in (2).

Proof. Since $f \in W_{\sigma}^{1}$ and $W_{\sigma}^{1} \subset W_{\sigma}^{2}$, by the Paley-Wiener Theorem this function is represented in the form (1), where $\varphi$ is determined by the equality (2). Furthermore, the series in (2) converges uniformly on every interval of the positive real semiaxis by the Weierstrass M-test. Then the series can be integrated term by term

$$
\begin{gathered}
\chi_{1}(z)=\frac{1}{\sqrt{2 \pi}} \int_{0}^{\sigma} \sum_{k=-\infty}^{+\infty} c_{k} e^{\frac{-i k \pi t}{\sigma}} e^{i t z} d t=\frac{1}{\sqrt{2 \pi}} \sum_{k=-\infty}^{+\infty} c_{k} \frac{e^{i \sigma\left(z-\frac{k \pi}{\sigma}\right)}-1}{i\left(z-\frac{k \pi}{\sigma}\right)}, \\
\chi_{2}(-i z)=-\frac{1}{\sqrt{2 \pi}} \int_{-\sigma}^{0} \sum_{k=-\infty}^{+\infty} c_{k} e^{\frac{-i k \pi t}{\sigma}} e^{t z} d t=-\frac{1}{\sqrt{2 \pi}} \sum_{k=-\infty}^{+\infty} c_{k} \frac{1-e^{-\sigma\left(z-i \frac{k \pi}{\sigma}\right)}}{\left(z-i \frac{k \pi}{\sigma}\right)} .
\end{gathered}
$$

Therefore

$$
\chi(z)=\chi_{1}(z)+i \chi_{2}(-i z)=\frac{1}{\sqrt{2 \pi}} \sum_{k=-\infty}^{+\infty} c_{k} i\left(\frac{1-e^{i \sigma\left(z-\frac{k \pi}{\sigma}\right)}}{z-\frac{k \pi}{\sigma}}-\frac{1-e^{-\sigma\left(z-i \frac{k \pi}{\sigma}\right)}}{z-i \frac{k \pi}{\sigma}}\right) .
$$

For $z=x-i \frac{\pi}{2 \sigma}$ we have

$$
\begin{aligned}
& \chi\left(x-i \frac{\pi}{2 \sigma}\right)=-\frac{1}{\sqrt{2 \pi}} \sum_{k=-\infty}^{+\infty} c_{k} \frac{(-1)^{k} i e^{i \sigma x+\frac{\pi}{2}}}{x-\frac{\pi}{\sigma}\left(\frac{i}{2}+k\right)}+\frac{1}{\sqrt{2 \pi}} \sum_{k=-\infty}^{+\infty} c_{k} \frac{(-1)^{k} i e^{-\sigma x+\frac{\pi i}{2}}}{x-i \frac{\pi}{\sigma}\left(\frac{1}{2}+k\right)}+ \\
& +\frac{1}{\sqrt{2 \pi}} \sum_{k=-\infty}^{+\infty} c_{k} \frac{\pi k(1+i)}{\sigma\left(x-\frac{\pi}{\sigma}\left(\frac{i}{2}+k\right)\right)\left(x-i \frac{\pi}{\sigma}\left(\frac{1}{2}+k\right)\right)}=-T_{1}(x)+T_{2}(x)+T_{3}(x) .
\end{aligned}
$$

Consider the integral over $x \in\left[\frac{\pi}{\sigma} ;+\infty\right)$ from the modulus of the first addend

$$
\begin{gathered}
\int_{\frac{\pi}{\sigma}}^{+\infty}\left|T_{1}(x)\right| d x=\sum_{n=1}^{+\infty} \int_{0}^{\frac{\pi}{\sigma}}\left|T_{1}\left(x+\frac{\pi n}{\sigma}\right)\right| d x= \\
=\frac{1}{\sqrt{2 \pi}} \sum_{n=1}^{+\infty} \int_{0}^{\frac{\pi}{\sigma}}\left|i e^{i \sigma\left(x+\frac{\pi n}{\sigma}\right)+\frac{\pi}{2}} \sum_{k=-\infty}^{+\infty} \frac{(-1)^{k} c_{k}}{x-\frac{\pi}{\sigma}\left(\frac{i}{2}+k\right)+\frac{\pi n}{\sigma}}\right| d x= \\
=\frac{e^{\frac{\pi}{2}}}{\sqrt{2 \pi}} \sum_{n=1}^{+\infty} \int_{0}^{\frac{\pi}{\sigma}}\left|\sum_{k=-\infty}^{+\infty} \frac{(-1)^{k} c_{k}}{x-\frac{\pi}{\sigma}\left(\frac{i}{2}+k\right)+\frac{\pi n}{\sigma}}\right| d x .
\end{gathered}
$$

Let $k^{\prime}=k-n$, then

$$
\int_{\frac{\pi}{\sigma}}^{+\infty}\left|T_{1}(x)\right| d x=\frac{e^{\frac{\pi}{2}}}{\sqrt{2 \pi}} \sum_{n=1}^{+\infty} \int_{0}^{\frac{\pi}{\sigma}}\left|\sum_{k^{\prime}=-\infty}^{+\infty} \frac{(-1)^{k^{\prime}+n} c_{k^{\prime}+n}}{x-\frac{i \pi}{2 \sigma}-\frac{\pi}{\sigma} k^{\prime}}\right| d x .
$$


Therefore

$$
\begin{aligned}
& \int_{\frac{\pi}{\sigma}}^{+\infty}\left|T_{1}(x)\right| d x=\frac{e^{\frac{\pi}{2}}}{\sqrt{2 \pi}}\left(\sum_{n=1}^{+\infty} \int_{0}^{\frac{\pi}{\sigma}} \mid \sum_{k^{\prime}=-\infty}^{+\infty} \frac{(-1)^{k^{\prime}+n} c_{k^{\prime}+n}}{x-\frac{i \pi}{2 \sigma}-\frac{\pi}{\sigma} k^{\prime}}-\sum_{k^{\prime}=-\infty}^{+\infty} \frac{(-1)^{k^{\prime}+n} c_{k^{\prime}+n}}{\frac{\pi}{\sigma}\left(-\frac{i}{2}-k^{\prime}\right)}+\right. \\
& \left.+\sum_{k^{\prime}=-\infty}^{+\infty} \frac{(-1)^{k^{\prime}+n} c_{k^{\prime}+n}}{\frac{\pi}{\sigma}\left(-\frac{i}{2}-k^{\prime}\right)}+\sum_{k^{\prime}=-\infty}^{+\infty} \frac{(-1)^{k^{\prime}+n} c_{k^{\prime}+n} k^{\prime}}{\frac{\pi}{\sigma}\left(1+k^{\prime 2}\right)}-\sum_{k^{\prime}=-\infty}^{+\infty} \frac{(-1)^{k^{\prime}+n} c_{k^{\prime}+n} k^{\prime}}{\frac{\pi}{\sigma}\left(1+k^{\prime 2}\right)} \mid d x\right) \leq \\
& \leq \frac{e^{\frac{\pi}{2}}}{\sqrt{2 \pi}} \sum_{n=1}^{+\infty}\left(\int_{0}^{\frac{\pi}{\sigma}}\left|\sum_{k^{\prime}=-\infty}^{+\infty}(-1)^{k^{\prime}+n} c_{k^{\prime}+n}\left(\frac{1}{x-\frac{i \pi}{2 \sigma}-\frac{\pi}{\sigma} k^{\prime}}-\frac{1}{\frac{i \pi}{2 \sigma}-\frac{\pi}{\sigma} k^{\prime}}\right)\right| d x+\right. \\
& +\int_{0}^{\frac{\pi}{\sigma}}\left|\sum_{k^{\prime}=-\infty}^{+\infty}(-1)^{k^{\prime}+n} c_{k^{\prime}+n}\left(\frac{1}{-\frac{i \pi}{2 \sigma}-\frac{\pi}{\sigma} k^{\prime}}+\frac{k^{\prime}}{\frac{\pi}{\sigma}+\frac{\pi}{\sigma} k^{\prime 2}}\right)\right| d x+ \\
& \left.+\int_{0}^{\frac{\pi}{\sigma}}\left|\sum_{k^{\prime}=-\infty}^{+\infty} \frac{(-1)^{k^{\prime}+n} c_{k^{\prime}+n} k^{\prime}}{\frac{\pi}{\sigma}\left(1+k^{\prime 2}\right)}\right| d x\right)=\frac{e^{-\frac{\pi}{2}}}{\sqrt{2 \pi}}\left(\sum_{n=1}^{+\infty} I_{1}(n)+\sum_{n=1}^{+\infty} I_{2}(n)+\sum_{n=1}^{+\infty} I_{3}(n)\right) .
\end{aligned}
$$

Consider the first sum. The series

$$
\sum_{k^{\prime}=-\infty}^{+\infty}(-1)^{k^{\prime}+n} c_{k^{\prime}+n}\left(\frac{1}{x-\frac{i \pi}{2 \sigma}-\frac{\pi}{\sigma} k^{\prime}}-\frac{1}{\frac{i \pi}{2 \sigma}-\frac{\pi}{\sigma} k^{\prime}}\right)
$$

converges uniformly on every bounded interval by the Weierstrass M-test. Therefore, term by term integration leads

$$
\begin{gathered}
\sum_{n=1}^{+\infty} I_{1}(n) \leq \sum_{n=1}^{+\infty} \int_{0}^{\frac{\pi}{\sigma}} \sum_{k^{\prime}=-\infty}^{+\infty}\left|c_{k^{\prime}+n}\right|\left|\frac{-x}{\left(x-\frac{i \pi}{2 \sigma}-\frac{\pi}{\sigma} k^{\prime}\right)\left(-\frac{i \pi}{2 \sigma}-\frac{\pi}{\sigma} k^{\prime}\right)}\right| d x \leq \\
\leq \sum_{n=1}^{+\infty} \sum_{k^{\prime}=-\infty}^{+\infty}\left|c_{k^{\prime}+n}\right| \int_{0}^{\frac{\pi}{\sigma}}\left|\frac{-x}{\left(x-\frac{i \pi}{2 \sigma}-\frac{\pi}{\sigma} k^{\prime}\right)\left(-\frac{i \pi}{2 \sigma}-\frac{\pi}{\sigma} k^{\prime}\right)}\right| d x .
\end{gathered}
$$

Then, applying the Mean-Value Theorem, we can write

$$
\int_{0}^{\frac{\pi}{\sigma}}\left|\frac{-x}{\left(x-\frac{i \pi}{2 \sigma}-\frac{\pi}{\sigma} k^{\prime}\right)\left(-\frac{i \pi}{2 \sigma}-\frac{\pi}{\sigma} k^{\prime}\right)}\right| d x=\frac{\alpha\left(\frac{\pi}{\sigma}\right)^{2}}{\left|\left(\frac{\alpha \pi}{\sigma}-\frac{i \pi}{2 \sigma}-\frac{\pi}{\sigma} k^{\prime}\right)\left(-\frac{i \pi}{2 \sigma}-\frac{\pi}{\sigma} k^{\prime}\right)\right|},
$$

where $\alpha \in[0 ; 1]$. Hence

$$
\sum_{n=1}^{+\infty} I_{1}(n) \leq \sum_{n=1}^{+\infty} \sum_{k^{\prime}=-\infty}^{+\infty}\left|c_{k^{\prime}+n}\right| \frac{\alpha}{\left|\left(\alpha-\frac{i}{2}-k^{\prime}\right)\left(-\frac{i}{2}-k^{\prime}\right)\right|} .
$$

On changing the order of summation of absolute convergent series we obtain

$$
\sum_{n=1}^{+\infty} I_{1}(n) \leq \sum_{k^{\prime}=-\infty}^{+\infty} \frac{1}{\sqrt{\left(\alpha-k^{\prime}\right)^{2}+\frac{1}{4}} \sqrt{k^{\prime 2}+\frac{1}{4}}} \sum_{n=1}^{+\infty}\left|c_{k^{\prime}+n}\right|
$$

Since

$$
\sum_{k^{\prime}=-\infty}^{+\infty} \frac{1}{\sqrt{\left(\alpha-k^{\prime}\right)^{2}+\frac{1}{4}} \sqrt{k^{\prime 2}+\frac{1}{4}}} \leq \sum_{k=-\infty}^{-1} \frac{1}{k^{\prime 2}+\frac{1}{4}}+b+\sum_{k=2}^{+\infty} \frac{1}{\left(k^{\prime}-1\right)^{2}+\frac{1}{4}}<+\infty
$$


and $\sum_{n=-\infty}^{+\infty}\left|c_{k^{\prime}+n}\right| \leq M<+\infty$, where the constant $M$ is independent of $k^{\prime}$, we have $\sum_{n=1}^{+\infty} I_{1}(n)<+\infty$.

We estimate $I_{2}(n)$. Let us remark that the integrand

$$
\left|\sum_{k^{\prime}=-\infty}^{+\infty}(-1)^{k^{\prime}+n} c_{k^{\prime}+n}\left(\frac{1}{-\frac{i \pi}{2 \sigma}-\frac{\pi}{\sigma} k^{\prime}}+\frac{k^{\prime}}{\frac{\pi}{\sigma}+\frac{\pi}{\sigma} k^{2}}\right)\right|
$$

is independent of $x$. On changing the order of summation we obtain

$$
\begin{aligned}
& \sum_{n=1}^{+\infty} I_{2}(n)=\sum_{n=1}^{+\infty} \int_{0}^{\frac{\pi}{\sigma}}\left|\sum_{k^{\prime}=-\infty}^{+\infty}(-1)^{k^{\prime}+n}\right| c_{k^{\prime}+n}\left|\frac{1-\frac{i}{2} k^{\prime}}{\frac{\pi}{\sigma}\left(-\frac{i}{2}-k^{\prime}\right)\left(1+k^{\prime 2}\right)}\right| d x \leq \\
\leq & \sum_{k^{\prime}=-\infty}^{+\infty} \frac{\sqrt{1+\frac{1}{4} k^{2}}}{\sqrt{k^{2}+\frac{1}{4}}\left(1+k^{\prime 2}\right)} \sum_{n=1}^{+\infty}\left|c_{k^{\prime}+n}\right| \leq 2 \sum_{k^{\prime}=-\infty}^{+\infty} \frac{1}{\left(1+k^{\prime 2}\right)} \sum_{n=-\infty}^{+\infty}\left|c_{k^{\prime}+n}\right|<+\infty .
\end{aligned}
$$

We now consider $I_{3}(n)$. The series is independent of $x$. Therefore, using Theorem B., we deduce $\sum_{n=1}^{+\infty} I_{3}(n)<+\infty$. Hence $\int_{\frac{\pi}{\sigma}}^{+\infty}\left|T_{1}(x)\right| d x<+\infty$.

Noting that

$$
\sum_{k=-\infty}^{+\infty} \frac{\left|c_{k}\right|}{\left|x-i \frac{\pi}{\sigma}\left(\frac{1}{2}+k\right)\right|} \leq \frac{1}{x} \sum_{k=-\infty}^{+\infty}\left|c_{k}\right|,
$$

we deduce $\int_{\frac{\pi}{\sigma}}^{+\infty}\left|T_{2}(x)\right| d x<+\infty$. Finally, inequality (6) implies $\int_{\frac{\pi}{\sigma}}^{+\infty}\left|T_{3}(x)\right| d x<+\infty$. The sufficiency is proved.

Now suppose that $f \in W_{\sigma}^{1}, \chi \in L^{1}\left(\frac{\pi}{\sigma} ;+\infty\right)$. We now prove that inequality (6) holds. Using (8), we conclude

$$
T_{3}(x)=\chi\left(x-\frac{i \pi}{2 \sigma}\right)+T_{1}(x)-T_{2}(x)
$$

As in the proof the first part of lemma we showe that $T_{1} \in L^{1}\left(\frac{\pi}{\sigma} ;+\infty\right)$ and $T_{2} \in L^{1}\left(\frac{\pi}{\sigma} ;+\infty\right)$. Hence $T_{3} \in L^{1}\left(\frac{\pi}{\sigma} ;+\infty\right)$. It is clear that inequality (6) holds.

Lemma 2. Let a function $f$ belong to $W_{\sigma}^{1}$. Then conditions (6) and (4) are equivalent.

Proof. Let the condition (4) holds. Then

$$
\begin{gathered}
\int_{\frac{\pi}{\sigma}}^{+\infty}\left|\sum_{k=-\infty}^{+\infty} c_{k} \frac{k}{\left(x-\frac{\pi}{\sigma}\left(\frac{i}{2}+k\right)\right)\left(x-i \frac{\pi}{\sigma}\left(\frac{1}{2}+k\right)\right)}\right| d x= \\
=\sum_{m=1}^{+\infty} \int_{0}^{\frac{\pi}{\sigma}}\left|\sum_{k=-\infty}^{+\infty} c_{k} \frac{k}{\left(x+\frac{\pi}{\sigma}\left(m-\frac{i}{2}-k\right)\right)\left(x+\frac{\pi}{\sigma}\left(m-\frac{i}{2}-i k\right)\right)}\right| d x= \\
=\sum_{m=1}^{+\infty} \int_{0}^{\frac{\pi}{\sigma}} \mid \sum_{k=-\infty}^{+\infty} c_{k} \frac{k}{\left(x+\frac{\pi}{\sigma}\left(m-\frac{i}{2}-k\right)\right)\left(x+\frac{\pi}{\sigma}\left(m-\frac{i}{2}-i k\right)\right)}- \\
-\sum_{k=-\infty}^{+\infty} c_{k} \frac{k}{\frac{\pi}{\sigma}\left(m-\frac{i}{2}-k\right) \frac{\pi}{\sigma}\left(m-\frac{i}{2}-i k\right)}+\sum_{k=-\infty}^{+\infty} c_{k} \frac{k}{\frac{\pi}{\sigma}\left(m-\frac{i}{2}-k\right) \frac{\pi}{\sigma}\left(m-\frac{i}{2}-i k\right)} \mid d x \leq
\end{gathered}
$$




$$
\begin{gathered}
\leq \sum_{m=1}^{+\infty}\left(\int_{0}^{\frac{\pi}{\sigma}} \mid \sum_{k=-\infty}^{+\infty} c_{k}\left(\frac{k}{\left(x+\frac{\pi}{\sigma}\left(m-\frac{i}{2}-k\right)\right)\left(x+\frac{\pi}{\sigma}\left(m-\frac{i}{2}-i k\right)\right)}-\right.\right. \\
\left.\left.-\frac{k}{\frac{\pi}{\sigma}\left(m-\frac{i}{2}-k\right) \frac{\pi}{\sigma}\left(m-\frac{i}{2}-i k\right)}\right)\left|d x+\frac{\pi}{\sigma}\right| \sum_{k=-\infty}^{+\infty} c_{k} \frac{k}{\frac{\pi}{\sigma}\left(m-\frac{i}{2}-k\right) \frac{\pi}{\sigma}\left(m-\frac{i}{2}-i k\right)} \mid\right) .
\end{gathered}
$$

Let us show that

$$
\sum_{m=1}^{+\infty} \int_{0}^{\frac{\pi}{\sigma}} \theta(m ; x) d x<+\infty
$$

where

$$
\begin{gathered}
\theta(m ; x)=\mid \sum_{k=-\infty}^{+\infty} c_{k}\left(\frac{k}{\left(x+\frac{\pi}{\sigma}\left(m-\frac{i}{2}-k\right)\right)\left(x+\frac{\pi}{\sigma}\left(m-\frac{i}{2}-i k\right)\right)}-\right. \\
\left.-\frac{k}{\frac{\pi}{\sigma}\left(m-\frac{i}{2}-k\right) \frac{\pi}{\sigma}\left(m-\frac{i}{2}-i k\right)}\right) \mid .
\end{gathered}
$$

Since

$$
\begin{gathered}
\frac{k}{\left(x+\frac{\pi}{\sigma}\left(m-\frac{i}{2}-k\right)\right)\left(x+\frac{\pi}{\sigma}\left(m-\frac{i}{2}-i k\right)\right)}= \\
=\frac{\sigma}{\pi(1-i)}\left(\frac{1}{x+\frac{\pi}{\sigma}\left(m-\frac{i}{2}-k\right)}-\frac{1}{\left(x+\frac{\pi}{\sigma}\left(m-\frac{i}{2}-i k\right)\right.}\right), \\
\frac{k}{\frac{\pi}{\sigma}\left(m-\frac{i}{2}-k\right) \frac{\pi}{\sigma}\left(m-\frac{i}{2}-i k\right)}=\frac{\sigma}{\pi(1-i)}\left(\frac{1}{\frac{\pi}{\sigma}\left(m-\frac{i}{2}-k\right)}-\frac{1}{\frac{\pi}{\sigma}\left(m-\frac{i}{2}-i k\right)}\right),
\end{gathered}
$$

we have

$$
\begin{gathered}
\theta(m ; x)=\mid \sum_{k=-\infty}^{+\infty} \frac{\sigma}{\pi(1-i)} c_{k}\left(\frac{-x}{\left(x+\frac{\pi}{\sigma}\left(m-\frac{i}{2}-k\right)\right) \frac{\pi}{\sigma}\left(m-\frac{i}{2}-k\right)}+\right. \\
\left.+\frac{x}{\left(x+\frac{\pi}{\sigma}\left(m-\frac{i}{2}-i k\right)\right) \frac{\pi}{\sigma}\left(m-\frac{i}{2}-i k\right)}\right) \mid d x \leq \\
\sum_{k=-\infty}^{+\infty} \frac{\sigma}{\sqrt{2} \pi}\left|c_{k}\right|\left(\left|\frac{-x}{\left(x+\frac{\pi}{\sigma}\left(m-\frac{i}{2}-k\right)\right) \frac{\pi}{\sigma}\left(m-\frac{i}{2}-k\right)}\right|+\right. \\
\int_{0}^{\frac{\pi}{\sigma}} \theta(m ; x) d x \leq \frac{1}{\sqrt{2}} \sum_{k=-\infty}^{+\infty}\left|c_{k}\right| \frac{x}{\sqrt{(\alpha-k+m)^{2}+\frac{1}{4}} \sqrt{(-k+m)^{2}+\frac{1}{4}}}+ \\
+\frac{1}{\sqrt{2}} \sum_{k=-\infty}^{+\infty}\left|c_{k}\right| \frac{\alpha}{\sqrt{(\alpha+m)^{2}+\left(k+\frac{1}{2}\right)^{2}} \sqrt{m^{2}+\left(k+\frac{1}{2}\right)^{2}}},
\end{gathered}
$$


where $\alpha \in[0 ; 1]$. In the first sum we change the order of summation and let $m^{\prime}=m-k$, that

$$
\begin{gathered}
\sum_{m=1}^{+\infty} \sum_{k=-\infty}^{+\infty}\left|c_{k}\right| \frac{\alpha}{\sqrt{(\alpha-k+m)^{2}+\frac{1}{4}} \sqrt{(-k+m)^{2}+\frac{1}{4}}} \leq \\
\leq \sum_{k=-\infty}^{+\infty}\left|c_{k}\right| \sum_{m^{\prime}=1-k}^{+\infty} \frac{\alpha}{\sqrt{\left(\alpha+m^{\prime}\right)^{2}+\frac{1}{4}} \sqrt{m^{\prime 2}+\frac{1}{4}}} \leq \\
\leq \sum_{k=-\infty}^{0}\left|c_{k}\right| \sum_{m^{\prime}=1-k}^{+\infty} \frac{1}{m^{\prime 2}+\frac{1}{4}}+\sum_{k=1}^{+\infty}\left|c_{k}\right| \sum_{m^{\prime}=1-k}^{+\infty} \frac{1}{\left(1+m^{\prime}\right)^{2}+\frac{1}{4}}<+\infty .
\end{gathered}
$$

Let us estimate the second sum in the right-hand side of (9). We note that

$$
\begin{gathered}
\sum_{k=-\infty}^{+\infty}\left|c_{k}\right| \frac{\alpha}{\sqrt{(\alpha+m)^{2}+\left(k+\frac{1}{2}\right)^{2}} \sqrt{m^{2}+\left(k+\frac{1}{2}\right)^{2}}} \leq \\
\leq \sum_{k=-\infty}^{+\infty}\left|c_{k}\right| \frac{1}{m^{2}+\left(k+\frac{1}{2}\right)^{2}} \leq \sum_{k=-\infty}^{+\infty}\left|c_{k}\right| \frac{1}{m^{2}} .
\end{gathered}
$$

It follows that

$$
\sum_{m=1}^{+\infty} \sum_{k=-\infty}^{+\infty}\left|c_{k}\right| \frac{\alpha}{\sqrt{(\alpha+m)^{2}+\left(k+\frac{1}{2}\right)^{2}} \sqrt{m^{2}+\left(k+\frac{1}{2}\right)^{2}}} \leq \sum_{k=-\infty}^{+\infty}\left|c_{k}\right| \sum_{m=1}^{+\infty} \frac{1}{m^{2}}<+\infty .
$$

Using this inequality we obtain

$$
\sum_{m=1}^{+\infty} \int_{0}^{\frac{\pi}{\sigma}} \theta(m ; x) d x<+\infty
$$

Finally, the condition (6) holds.

Let now the condition (6) holds. Then

$$
\begin{gathered}
\sum_{m=1}^{+\infty}\left|\sum_{k=-\infty}^{+\infty} c_{k} \frac{k}{\frac{\pi}{\sigma}\left(m-\frac{i}{2}-k\right) \frac{\pi}{\sigma}\left(m-\frac{i}{2}-i k\right)}\right|=\sum_{m=1}^{+\infty} \mid \sum_{k=-\infty}^{+\infty} c_{k} \frac{k}{\frac{\pi}{\sigma}\left(m-\frac{i}{2}-k\right) \frac{\pi}{\sigma}\left(m-\frac{i}{2}-i k\right)}- \\
-\int_{0}^{\frac{\pi}{\sigma}} \sum_{k=-\infty}^{+\infty} c_{k} \frac{k}{\left(x+\frac{\pi}{\sigma}\left(m-\frac{i}{2}-k\right)\right)\left(x+i \frac{\pi}{\sigma}\left(m-\frac{1}{2}-k\right)\right)} d x+ \\
+\int_{0}^{\frac{\pi}{\sigma}} \sum_{k=-\infty}^{+\infty} c_{k} \frac{k}{\left(x+\frac{\pi}{\sigma}\left(m-\frac{i}{2}-k\right)\right)\left(x+i \frac{\pi}{\sigma}\left(m-\frac{1}{2}-k\right)\right)} d x \mid \leq \\
\leq \sum_{m=1}^{+\infty} \int_{0}^{\frac{\pi}{\sigma}} \mid \sum_{k=-\infty}^{+\infty} c_{k}\left(\frac{k}{\frac{\pi}{\sigma}\left(m-\frac{i}{2}-k\right) \frac{\pi}{\sigma}\left(m-\frac{i}{2}-i k\right)}-\right. \\
\left.-\frac{k}{\left(x+\frac{\pi}{\sigma}\left(m-\frac{i}{2}-k\right)\right)\left(x+i \frac{\pi}{\sigma}\left(m-\frac{1}{2}-k\right)\right)}\right) \mid d x+
\end{gathered}
$$




$$
+\sum_{m=1}^{+\infty} \int_{0}^{\frac{\pi}{\sigma}}\left|\sum_{k=-\infty}^{+\infty} c_{k} \frac{k}{\left(x+\frac{\pi}{\sigma}\left(m-\frac{i}{2}-k\right)\right)\left(x+i \frac{\pi}{\sigma}\left(m-\frac{1}{2}-k\right)\right)}\right| d x .
$$

As in the proof the first part of the lemma we show that

$$
\sum_{m=1}^{+\infty} \int_{0}^{\frac{\pi}{\sigma}} \theta(m ; x) d x<+\infty
$$

and the proof is complete.

Lemma 3. Let $f \in W_{\sigma}^{1}$. Then $\chi\left(i y-\frac{\pi}{2 \sigma}\right) \in L^{1}\left(\frac{\pi}{\sigma} ;+\infty\right)$ defined by (3) if and only if

$$
\int_{\frac{\pi}{\sigma}}^{+\infty}\left|\sum_{k=-\infty}^{+\infty} c_{k} \frac{k}{\left(y+i \frac{\pi}{\sigma}\left(k+\frac{1}{2}\right)\right)\left(y+\frac{\pi}{\sigma}\left(\frac{i}{2}-k\right)\right)}\right| d y<+\infty,
$$

where coefficients $c_{k}$ defined by (2).

Proof. Since $f \in W_{\sigma}^{1}, \chi$ is determined by equality (7). For $z=i y-\frac{\pi}{2 \sigma}$ we have

$$
\begin{aligned}
& \chi\left(i y-\frac{\pi}{2 \sigma}\right)=\frac{1}{\sqrt{2 \pi}} \sum_{k=-\infty}^{+\infty} c_{k} \frac{(-1)^{k} i e^{-\sigma y}}{y+i \frac{\pi}{\sigma}\left(k+\frac{1}{2}\right)}+\frac{1}{\sqrt{2 \pi}} \sum_{k=-\infty}^{+\infty} c_{k} \frac{(-1)^{k} e^{-\sigma i y+\frac{\pi}{2}}}{y+\frac{\pi}{\sigma}\left(\frac{i}{2}-k\right)}+ \\
& +\frac{1}{\sqrt{2 \pi}} \sum_{k=-\infty}^{+\infty} c_{k} \frac{\pi k(-1-i)}{\sigma\left(y+i \frac{\pi}{\sigma}\left(k+\frac{1}{2}\right)\right)\left(y+\frac{\pi}{\sigma}\left(\frac{i}{2}-k\right)\right)}=F_{1}(y)+F_{2}(y)+F_{3}(y) .
\end{aligned}
$$

Noting that

$$
\sum_{k=-\infty}^{+\infty} \frac{\left|c_{k}\right|}{\left|y+i \frac{\pi}{\sigma}\left(k+\frac{1}{2}\right)\right|} \leq \frac{1}{y} \sum_{k=-\infty}^{+\infty}\left|c_{k}\right|
$$

we obtain $\int_{\frac{\pi}{\sigma}}^{+\infty}\left|F_{1}(y)\right| d y<+\infty$. Consider the integral over $y \in\left[\frac{\pi}{\sigma} ;+\infty\right)$ of modulus of $F_{2}$

$$
\begin{gathered}
\int_{\frac{\pi}{\sigma}}^{+\infty}\left|F_{2}(y)\right| d y=\sum_{n=1}^{+\infty} \int_{0}^{\frac{\pi}{\sigma}}\left|F_{2}\left(y+\frac{\pi n}{\sigma}\right)\right| d y= \\
=\frac{1}{\sqrt{2 \pi}} \sum_{n=1}^{+\infty} \int_{0}^{\frac{\pi}{\sigma}}\left|e^{-i \sigma\left(y+\frac{\pi n}{\sigma}\right)+\frac{\pi}{2}} \sum_{k=-\infty}^{+\infty} \frac{(-1)^{k} c_{k}}{y+\frac{\pi n}{\sigma}+\frac{\pi}{\sigma}\left(\frac{i}{2}-k\right)}\right| d y \leq \\
\leq \frac{e^{\frac{\pi}{2}}}{\sqrt{2 \pi}} \sum_{n=1}^{+\infty} \int_{0}^{\frac{\pi}{\sigma}}\left|\sum_{k=-\infty}^{+\infty} \frac{(-1)^{k} c_{k}}{y+\frac{\pi n}{\sigma}+\frac{\pi}{\sigma}\left(\frac{i}{2}-k\right)}\right| d y .
\end{gathered}
$$

We use the estimation of $T_{1}$ from Lemma 1 and deduce $\int_{\frac{\pi}{\sigma}}^{+\infty}\left|F_{2}(y)\right| d y<+\infty$. We note that from inequality (10) it follows that $\int_{\frac{\pi}{\alpha}}^{+\infty}\left|F_{3}(y)\right| d y<+\infty$.

Now suppose that $\chi\left(i y-\frac{\pi}{2 \sigma}\right) \in L^{1}\left(\frac{\pi}{\sigma} ;+\infty\right)$. Let us show that inequality (10) holds. Then (11) implies

$$
F_{3}(y)=\chi\left(y-\frac{\pi}{2 \sigma}\right)+F_{1}(y)-F_{2}(y)
$$

As in the proof the first part of the lemma we show that $F_{1} \in L^{1}\left(\frac{\pi}{\sigma} ;+\infty\right)$ and $F_{2} \in L^{1}\left(\frac{\pi}{\sigma} ;+\infty\right)$ without using (10). Therefore $F_{3} \in L^{1}\left(\frac{\pi}{\sigma} ;+\infty\right)$. It is obvious that (10) holds. 
Lemma 4. Let a function $f$ belong to $W_{\sigma}^{1}$. Then (10) is equivalent to (5).

Proof. Let the inequality (5) hold. Then

$$
\begin{gathered}
\int_{\frac{\pi}{\sigma}}^{+\infty}\left|\sum_{k=-\infty}^{+\infty} c_{k} \frac{k}{\left(y+i \frac{\pi}{\sigma}\left(k+\frac{1}{2}\right)\right)\left(y+\frac{\pi}{\sigma}\left(\frac{i}{2}-k\right)\right)}\right| d y= \\
=\sum_{m=1}^{+\infty} \int_{0}^{\frac{\pi}{\sigma}}\left|\sum_{k=-\infty}^{+\infty} c_{k} \frac{k}{\left(y+\frac{\pi}{\sigma}\left(m+\frac{i}{2}+i k\right)\right)\left(y+\frac{\pi}{\sigma}\left(m-k+\frac{i}{2}\right)\right)}\right| d y= \\
=\sum_{m=1}^{+\infty} \int_{0}^{\frac{\pi}{\sigma}} \mid \sum_{k=-\infty}^{+\infty} c_{k} \frac{k}{\left(y+\frac{\pi}{\sigma}\left(m+\frac{i}{2}+i k\right)\right)\left(y+\frac{\pi}{\sigma}\left(m-k+\frac{i}{2}\right)\right)}- \\
-\sum_{k=-\infty}^{+\infty} c_{k} \frac{k}{\frac{\pi}{\sigma}\left(m+\frac{i}{2}+i k\right) \frac{\pi}{\sigma}\left(m-k+\frac{i}{2}\right)}+\sum_{k=-\infty}^{+\infty} c_{k} \frac{k}{\frac{\pi}{\sigma}\left(m+\frac{i}{2}+i k\right) \frac{\pi}{\sigma}\left(m-k+\frac{i}{2}\right)} \mid d y \leq \\
\leq \sum_{m=1}^{+\infty}\left(\int_{0}^{\frac{\pi}{\sigma}} \mid \sum_{k=-\infty}^{+\infty} c_{k}\left(\frac{k}{\left(y+\frac{\pi}{\sigma}\left(m+\frac{i}{2}+i k\right)\right)\left(y+\frac{\pi}{\sigma}\left(m-k+\frac{i}{2}\right)\right)}-\right.\right. \\
\left.\left.-\frac{k}{\frac{\pi}{\sigma}\left(m+\frac{i}{2}+i k\right) \frac{\pi}{\sigma}\left(m-k+\frac{i}{2}\right)}\right)\left|d y+\frac{\pi}{\sigma}\right| \sum_{k=-\infty}^{+\infty} c_{k} \frac{k}{\frac{\pi}{\sigma}\left(m+\frac{i}{2}+i k\right) \frac{\pi}{\sigma}\left(m-k+\frac{i}{2}\right)} \mid\right)
\end{gathered}
$$

Let us prove that

$$
\sum_{m=1}^{+\infty} \int_{0}^{\frac{\pi}{\sigma}} \vartheta(m ; y) d y<+\infty
$$

where

$$
\begin{gathered}
\vartheta(m ; y)=\mid \sum_{k=-\infty}^{+\infty} c_{k}\left(\frac{k}{\left(y+\frac{\pi}{\sigma}\left(m+\frac{i}{2}+i k\right)\right)\left(y+\frac{\pi}{\sigma}\left(m-k+\frac{i}{2}\right)\right)}-\right. \\
\left.-\frac{k}{\frac{\pi}{\sigma}\left(m+\frac{i}{2}+i k\right) \frac{\pi}{\sigma}\left(m-k+\frac{i}{2}\right)}\right) \mid
\end{gathered}
$$

Since

$$
\begin{gathered}
\frac{k}{\left(y+\frac{\pi}{\sigma}\left(m+\frac{i}{2}+i k\right)\right)\left(y+\frac{\pi}{\sigma}\left(m-k+\frac{i}{2}\right)\right)}= \\
=\frac{\sigma}{\pi(-1-i)}\left(\frac{1}{y+\frac{\pi}{\sigma}\left(m+\frac{i}{2}+k i\right)}-\frac{1}{y+\frac{\pi}{\sigma}\left(m-k+\frac{i}{2}\right)}\right) \\
\frac{k}{\frac{\pi}{\sigma}\left(m+\frac{i}{2}+i k\right) \frac{\pi}{\sigma}\left(m-k+\frac{i}{2}\right)}=\frac{\sigma}{\pi(-1-i)}\left(\frac{1}{\frac{\pi}{\sigma}\left(m+\frac{i}{2}+k i\right)}-\frac{1}{\frac{\pi}{\sigma}\left(m-k+\frac{i}{2}\right)}\right)
\end{gathered}
$$

we deduce

$$
\vartheta(k ; y)=\mid \sum_{k=-\infty}^{+\infty} \frac{\sigma}{\pi(-1-i)} c_{k}\left(\frac{-y}{\left(y+\frac{\pi}{\sigma}\left(m+k i+\frac{i}{2}\right)\right) \frac{\pi}{\sigma}\left(m+\frac{i}{2}+i k\right)}+\right.
$$




$$
\begin{gathered}
\left.+\frac{y}{\left(y+\frac{\pi}{\sigma}\left(m-k+\frac{i}{2}\right)\right) \frac{\pi}{\sigma}\left(m-k+\frac{i}{2}\right)}\right) \mid \leq \\
\leq \sum_{k=-\infty}^{+\infty} \frac{\sigma}{\sqrt{2} \pi}\left|c_{k}\right|\left(\left|\frac{-y}{\left(y+\frac{\pi}{\sigma}\left(m+k i+\frac{i}{2}\right)\right) \frac{\pi}{\sigma}\left(m+\frac{i}{2}+i k\right)}\right|+\right. \\
\left.+\left|\frac{y}{\left(y+\frac{\pi}{\sigma}\left(m-k+\frac{i}{2}\right)\right) \frac{\pi}{\sigma}\left(m-k+\frac{i}{2}\right)}\right|\right) .
\end{gathered}
$$

Using the mean value theorem, we get

$$
\begin{aligned}
& \int_{0}^{\frac{\pi}{\sigma}} \vartheta(k ; y) d y \leq \frac{1}{\sqrt{2}} \sum_{k=-\infty}^{+\infty}\left|c_{k}\right| \frac{\alpha}{\sqrt{(\alpha+m)^{2}+\left(k+\frac{1}{2}\right)^{2}} \sqrt{m^{2}+\left(k+\frac{1}{2}\right)^{2}}}+ \\
&+\frac{1}{\sqrt{2}} \sum_{k=-\infty}^{+\infty}\left|c_{k}\right| \frac{\alpha}{\sqrt{(\alpha-k+m)^{2}+\frac{1}{4}} \sqrt{(m-k)^{2}+\frac{1}{4}}}
\end{aligned}
$$

where $\alpha \in[0 ; 1]$. As in the proof of Lemma 2 we now conclude that these series are convergent. Therefore, we find that (12) hold. Thus, (10) holds.

Let now the condition (10) holds. Then

$$
\begin{gathered}
\sum_{m=1}^{+\infty}\left|\sum_{k=-\infty}^{+\infty} c_{k} \frac{k}{\frac{\pi}{\sigma}\left(m+\frac{i}{2}+i k\right) \frac{\pi}{\sigma}\left(m-k+\frac{i}{2}\right)}\right|= \\
=\sum_{m=1}^{+\infty} \mid \sum_{k=-\infty}^{+\infty} c_{k} \frac{k}{\frac{\pi}{\sigma}\left(m+\frac{i}{2}+i k\right) \frac{\pi}{\sigma}\left(m-k+\frac{i}{2}\right)}- \\
-\int_{0}^{\frac{\pi}{\sigma}} \sum_{k=-\infty}^{+\infty} c_{k} \frac{k}{\left(y+\frac{\pi}{\sigma}\left(m+\frac{i}{2}+i k\right)\right)\left(y+\frac{\pi}{\sigma}\left(m-k+\frac{i}{2}\right)\right)} d y+ \\
+\int_{0}^{\frac{\pi}{\sigma}} \sum_{k=-\infty}^{+\infty} c_{k} \frac{k}{\left(y+\frac{\pi}{\sigma}\left(m+\frac{i}{2}+i k\right)\right)\left(y+\frac{\pi}{\sigma}\left(m-k+\frac{i}{2}\right)\right)} d y \mid \leq \\
\leq \sum_{m=1}^{+\infty} \int_{0}^{\frac{\pi}{\sigma}} \vartheta(m ; y) d y+\int_{\frac{\pi}{\sigma}}^{+\infty}\left|\sum_{k=-\infty}^{+\infty} c_{k} \frac{k}{\left(y+\frac{\pi}{\sigma}\left(\frac{i}{2}+i k\right)\right)\left(y+\frac{\pi}{\sigma}\left(\frac{i}{2}-k\right)\right)}\right| d y .
\end{gathered}
$$

As in the proof the first part of the lemma we deduce that (12) holds without using (5). Then, inequality (5) is proved.

We need the following lemma (see [11], [12]).

Lemma 5. If a function $\chi$ is analytic in $\mathbb{C}(\alpha ; \beta)$, has the angle boundary values a. e. on $\partial \mathbb{C}(\alpha ; \beta), \chi \in L^{p}(\partial \mathbb{C}(\alpha ; \beta))$, and for some $\gamma \in(0 ; \pi /(\alpha-\beta))$

$$
(\forall \varepsilon>0): \sup _{\alpha<\varphi<\beta}\left\{\int_{0}^{+\infty}\left|\chi\left(r e^{i \varphi}\right)\right|^{p} e^{-\varepsilon r^{\gamma}} d r\right\}^{\frac{1}{p}}<+\infty,
$$

then $\chi \in E^{p}[\mathbb{C}(\alpha ; \beta)]$. 
Proof of Theorems 1. Let $f \in W_{\sigma}^{1}$ admits splitting $f=\chi+\mu$, where $\chi$ and $\mu$ are analytic in $\mathbb{C}_{+}$. Further, $\chi \in E^{1}\left[\mathbb{C}\left(0 ; \frac{\pi}{2}\right)\right], \mu \in E^{1}\left[\mathbb{C}\left(-\frac{\pi}{2} ; 0\right)\right]$. Let us remark that $\chi \in E^{1}\left[\mathbb{C}\left(0 ; \frac{\pi}{2}\right)\right]$ implies $\chi\left(z-\frac{i \pi}{2 \sigma}\right) \in E^{1}\left[\mathbb{C}\left(0 ; \frac{\pi}{2}\right)\right]([14])$. Therefore, $\chi\left(x-\frac{i \pi}{2 \sigma}\right) \in L^{1}\left(\partial \mathbb{C}\left(0 ; \frac{\pi}{2}\right)\right)$. Thus we have $\chi\left(x-\frac{i \pi}{2 \sigma}\right) \in L^{1}\left(\frac{\pi}{\sigma} ;+\infty\right)$. We note that from Lemmas 1 and 2 it follows that condition (4) holds. Similarly, it can be shown that $\chi\left(i y-\frac{\pi}{2 \sigma}\right) \in L^{1}\left(\frac{\pi}{\sigma} ;+\infty\right)$. Since it is evident that Lemmas 3 and 4 implies (5), this proves the necessity.

Let $f \in W_{\sigma}^{1}$ and conditions (4), (5) hold. From Lemmas 1-4, we get the following $\chi(x-$ $\left.\frac{i \pi}{2 \sigma}\right) \in L^{1}\left(\frac{\pi}{\sigma} ;+\infty\right)$ and $\chi\left(i y-\frac{\pi}{2 \sigma}\right) \in L^{1}\left(\frac{\pi}{\sigma} ;+\infty\right)$. Notice that $\chi$ is an entire function yields $\chi\left(z-\frac{i \pi}{2 \sigma}-\frac{\pi}{2 \sigma}\right) \in L^{1}\left(\partial \mathbb{C}\left(0 ; \frac{\pi}{2}\right)\right)$. Since $\chi \in W_{\sigma}^{2}$ by the Paley-Wiener Theorem, for any $\varepsilon>0$ we obtain

$$
\begin{gathered}
\int_{0}^{+\infty}\left|\chi\left(r e^{i \varphi}-\frac{i \pi}{2 \sigma}-\frac{\pi}{2 \sigma}\right)\right| e^{-\varepsilon r^{\frac{3}{2}}} d r= \\
=\int_{0}^{+\infty}\left|\chi\left(r e^{i \varphi}-\frac{i \pi}{2 \sigma}-\frac{\pi}{2 \sigma}\right)\right| e^{-r \sigma|\sin \varphi|} e^{-\varepsilon r^{\frac{3}{2}}+r \sigma \sin \varphi} d r \leq \\
\leq\left(\int_{0}^{+\infty}\left|\chi\left(r e^{i \varphi}-\frac{i \pi}{2 \sigma}-\frac{\pi}{2 \sigma}\right)\right|^{2} e^{-2 r \sigma|\sin \varphi|} d r \int_{0}^{+\infty} e^{-\varepsilon r^{\frac{3}{2}}+r \sigma} d r\right)^{\frac{1}{2}} \leq b<+\infty,
\end{gathered}
$$

where $b$ is independent of $\varphi$. It follows from Lemma 5 that $\chi\left(z-\frac{i \pi}{2 \sigma}-\frac{\pi}{2 \sigma}\right) \in E^{1}\left[\mathbb{C}\left(0 ; \frac{\pi}{2}\right)\right]$.

To prove the theorem, we consider the space $\tilde{E}^{p}\left[\mathbb{C}\left(0 ; \frac{\pi}{2}\right)\right], 1 \leq p<+\infty$, of functions analytic in $\mathbb{C}_{+}$such that

$$
\max \left\{\sup _{y \in \mathbb{R}}\left\{\int_{0}^{+\infty}|f(x+i y)|^{p} e^{-p \sigma|y|} d x\right\} ; \sup _{x>0}\left\{\int_{-\infty}^{+\infty}|f(x+i y)|^{p} e^{-p \sigma|y|} d y\right\}\right\}^{\frac{1}{p}}<+\infty .
$$

We note that from Lemmas 2 in [13] it follows that $\chi\left(z-\frac{i \pi}{2 \sigma}-\frac{\pi}{2 \sigma}\right) \in \tilde{E}^{1}\left[\mathbb{C}\left(0 ; \frac{\pi}{2}\right)\right]$. Therefore, $\chi \in \tilde{E}^{1}\left[\mathbb{C}\left(0 ; \frac{\pi}{2}\right)\right]$. Finally, we apply Lemma 1 from $[13]$ and conclude that $\chi \in E^{1}\left[\mathbb{C}\left(0 ; \frac{\pi}{2}\right)\right]$. Similarly, it can be shown that $\mu \in E^{1}\left[\mathbb{C}\left(-\frac{\pi}{2} ; 0\right)\right]$.

\section{REFERENCES}

1. R.S. Yulmukhametov Splitting entire functions with zeros in a strip, Sbornik: Mathematics, 186 (1995), 1071-1084. (in Russian)

2. R.S. Yulmukhametov Solution of the Ehrenpreis factorization problem, Sbornik: Mathematics, 190 (1999), 597-629. (in Russian)

3. Yu.I. Lyubarskii Representation of functions in $H^{p}$ on half-plane and some applications, Teor. Funktsii, Funkts. Anal. Pril., 38 (1982), 76-84. (in Russian)

4. I.E. Chyzhykov Growth of pth means of analytic and subharmonic functions in the unit disc and angular distribution of zeros, http://arxiv.org/pdf/1509.02141.pdf, (2015).

5. V. Dilnyi Splitting of some spaces of analytic functions, Ufa Mathematical Journal, 6 (2014), 25-34.

6. B.J. Levin, Ju.I. Ljubarskii Interpolation by means of special classes of entire functions and related expanstions in series of exponentials, Mathematics of the USSR-Izvestiya, 9 (1975), 621-662. (in Russian)

7. G.Z. Ber On interferention phenomenon in integral metric and approximation of entire functions of exponential type, Teor. Funktsii, Funkts. Anal. Pril., 34 (1980), 11-24.

8. R. Boas, Entire functions, New York: Academic Press, 1954.

9. M.M. Djrbashian, Integral transforms and representations of functions in the complex domain, M: Sience, 1976. (in Russian) 
10. B.V. Vynnytskyi, V.M. Dilnyi On an analogue of Paley-Wiener's theorem for weighted Hardy spaces, Mat. Stud., 14 (2000), 35-40.

11. B.V. Vynnyts'kyi, V.M. Dil'nyi On solutions of homogeneous convolution equation generated by singularity, Mat. Stud., 19 (2003), 149-155.

12. V.M. Martirosian On a theorem of Djrbasian of the Phraugmen-Lindelof type, Math. Nachr., 144 (1989), 21-27.

13. V.M. Dilnyi Equivalent definition of some weighted Hardy spaces, Ukrainian Mathematical Journal, 60 (2008), 1477-1482.

Drohobych State Pedagogical University

dilnyi@ukr.net

taniosered@mail.ru 